\title{
Electron-electron scattering in linear transport in two-dimensional systems
}

\author{
Hu, Ben Yu-Kuang; Flensberg, Karsten
}

Published in:

Physical Review B Condensed Matter

Link to article, DOI:

10.1103/PhysRevB.53.10072

Publication date:

1996

Document Version

Publisher's PDF, also known as Version of record

Link back to DTU Orbit

Citation (APA):

Hu, B. Y-K., \& Flensberg, K. (1996). Electron-electron scattering in linear transport in two-dimensional systems. Physical Review B Condensed Matter, 53(5), 10072-10077. https://doi.org/10.1103/PhysRevB.53.10072

\section{General rights}

Copyright and moral rights for the publications made accessible in the public portal are retained by the authors and/or other copyright owners and it is a condition of accessing publications that users recognise and abide by the legal requirements associated with these rights.

- Users may download and print one copy of any publication from the public portal for the purpose of private study or research.

- You may not further distribute the material or use it for any profit-making activity or commercial gain

- You may freely distribute the URL identifying the publication in the public portal 


\title{
Electron-electron scattering in linear transport in two-dimensional systems
}

\author{
Ben Yu-Kuang $\mathrm{Hu}^{*}$ \\ Mikroelektronik Centret, Bygning 345 Фst, Danmarks Tekniske Universitet, DK-2800 Lyngby, Denmark \\ Karsten Flensberg ${ }^{\dagger}$ \\ Mikroelektronik Centret, Danmarks Tekniske Universitet, DK-2800 Lyngby, Denmark \\ and Dansk Institut for Fundamental Metrologi, Bygning 307, Anker Engelunds Vej 1, DK-2800 Lyngby, Denmark
}

(Received 10 October 1995)

\begin{abstract}
We describe a method for numerically incorporating electron-electron scattering in quantum wells for small deviations of the distribution function from equilibrium, within the framework of the Boltzmann equation. For a given temperature $T$ and density $n$, a symmetric matrix needs to be evaluated only once, and henceforth it can be used to describe electron-electron scattering in any Boltzmann equation linear-response calculation for that particular $T$ and $n$. Using this method, we calculate the distribution function and mobility for electrons in a quantum well, including full finite-temperature dynamic screening effects. We find that at some parameters that we investigated, electron-electron scattering reduces the mobility by approximately $40 \%$.
\end{abstract}

\section{INTRODUCTION}

The effect of electron-electron $(e-e)$ interactions on transport in bulklike systems of various dimensionalities is still an area of active research to this day, both experimentally ${ }^{1,2}$ and theoretically. ${ }^{3,4}$ On the face of it, it would seem that in the case of doped parabolic band semiconductors where umklapp processes are negligible, $e-e$ scattering should not affect the linear transport properties of bulklike systems (purely quantum effects such as weak-localization corrections excepted) since an $e-e$ scattering event conserves the total current in the system. Nevertheless, it has been appreciated for a long time that $e-e$ scattering can affect the mobility of a system semiclassically by scattering carriers into or out of parts of the Brillouin zone that are strongly affected by the other available scattering mechanisms. ${ }^{5,6}$

In Ref. 5, it was shown that the expression for mobility in the presence of quasielastic scatters takes on different forms in the limits of zero and infinitely strong $e-e$ scattering. Given a quasielastic energy-dependent transport (i.e., calculated with the $1-\cos \theta$ term) scattering time $\tau(\varepsilon)$ due to other scattering processes in the system such as acoustic phonons or impurities, the mobilities of the system for the cases of zero and infinite $e-e$ scattering rates, respectively, are given by

$$
\mu_{0}=\frac{e\langle\tau\rangle}{m} ; \quad \mu_{\infty}=\frac{e}{\left\langle\tau^{-1}\right\rangle m} .
$$

Here,

$$
\langle A\rangle=\frac{2}{n} \int \frac{d \mathbf{k}}{(2 \pi)^{d}} \varepsilon(\mathbf{k})\left(-\frac{\partial f_{0}}{\partial \varepsilon}\right) A(\mathbf{k}),
$$

where $n$ is the carrier density of the system, $\varepsilon(\mathbf{k})$ $=\hbar^{2} k^{2} /(2 m), f_{0}(\varepsilon(\mathbf{k}))$ is the Fermi-Dirac distribution function, $d$ is the dimensionality of the system, and we are assuming an isotropic parabolic band system. Clearly, for the case when that temperature $T$ is small on the scale of the energy scale over which $\tau(\varepsilon)$ varies significantly, $\langle\tau\rangle \approx\left[\left\langle\tau^{-1}\right\rangle\right]^{-1}$. Conversely, in the case where $T$ is large on the scale over which $\tau(\varepsilon)$ varies, there can be significant differences in the calculated mobility using the two different methods. For example, we show below that in particular cases in GaAs quantum wells, $\mu_{0} / \mu_{\infty} \approx 2$. Thus, for accurate theoretical determination of the mobility at the semiclassical level, it is important that $e-e$ scattering effects are included. Furthermore, it has been shown ${ }^{7}$ that experiments measuring the drag rate between electron gases between two coupled quantum wells are sensitive to the exact details of the linearresponse distribution function $f$ in each layer. Since $f$ is strongly affected by $e-e$ scattering in the intermediate temperature regime $T \approx 0.5 T_{F}$ (where $T_{F}$ is the Fermi temperature), it is important to include the effects of $e-e$ scattering in calculations of the drag rate.

In this paper, we demonstrate an efficient way of including $e-e$ scattering in the calculation of linear transport for two-dimensional (2D) cylindrically symmetric systems, within the semiclassical Boltzmann equation formalism. Similar calculations have been presented for linear transport in three dimensions ${ }^{3}$ and for relaxation properties of isotropic nonequilibrium distributions in two dimensions. ${ }^{8}$ Within this formalism, the Boltzmann equation for linear response can be solved exactly (within numerical accuracy). We have included full effects of finite-temperature dynamical screening, which automatically includes phenomena such as Landau damping and collective mode enhancements to scattering. The description of the $e-e$ scattering formalism for the Boltzmann equation is given in Secs. II and III, and Sec. IV contains the results and discussion. Throughout this paper, we assume that bands are isotropic and parabolic.

\section{ELECTRON-ELECTRON SCATTERING PROBABILITY}

The $e-e$ scattering occurs in the presence of other conduction electrons, and hence the bare interparticle Coulomb interaction $U(q)$ is screened. Furthermore, at the intermediate temperatures in which we are interested, the energy transfer 
between the electrons in a scattering event is often a substantial fraction of the kinetic energy of the electrons, and hence the scattering matrix elements for $e-e$ interactions should be calculated using the dynamically screened Coulomb interaction $V(q, \omega)=U(q) / \epsilon(q, \omega)$, where $\epsilon(q, \omega)$ is the dielectric function. In this paper, we use $\epsilon(q, \omega)$ given by the randomphase approximation (RPA), which we evaluate using a method described previously by $\mathrm{us}^{7}$ and we use the Born approximation for the scattering probability.

The scattering probability $w\left(\mathbf{k}_{1}^{\prime} \sigma_{1}, \mathbf{k}_{2}^{\prime} \sigma_{2} ; \mathbf{k}_{1} \sigma_{1}, \mathbf{k}_{2} \sigma_{2}\right)$ for a pair of electrons initially in states $\mathbf{k}_{1} \sigma_{1}, \mathbf{k}_{2} \sigma_{2}$ to be scattered to $\mathbf{k}_{1}^{\prime} \sigma_{1}, \mathbf{k}_{2}^{\prime} \sigma_{2}$ depends on whether or not the electrons have the same or opposite spins. For electrons with the same spin, say $\uparrow$,

$$
\begin{aligned}
w(\mathbf{k} & \left.+\mathbf{q} \uparrow, \mathbf{k}^{\prime}-\mathbf{q} \uparrow ; \mathbf{k} \uparrow, \mathbf{k}^{\prime} \uparrow\right) \\
& =\frac{1}{2} \frac{2 \pi}{\hbar}\left|V\left(\mathbf{q}, \varepsilon_{\mathbf{k}+\mathbf{q}}-\varepsilon_{\mathbf{k}}\right)-V\left(\mathbf{k}^{\prime}-\mathbf{q}+\mathbf{k}, \varepsilon_{\mathbf{k}^{\prime}-\mathbf{q}}-\varepsilon_{\mathbf{k}}\right)\right|^{2} .
\end{aligned}
$$

The fraction $1 / 2$ in Eq. (3) is due to double counting, since $w\left(\mathbf{k}+\mathbf{q} \uparrow, \mathbf{k}^{\prime}-\mathbf{q} \uparrow ; \mathbf{k} \uparrow, \mathbf{k}^{\prime} \uparrow\right)$ and $w\left(\mathbf{k}^{\prime}-\mathbf{q} \uparrow, \mathbf{k}+\mathbf{q} \uparrow ; \mathbf{k} \uparrow, \mathbf{k}^{\prime} \uparrow\right)$ describe exactly the same process. For opposite spins,

$$
w\left(\mathbf{k}+\mathbf{q} \uparrow, \mathbf{k}^{\prime}-\mathbf{q} \downarrow ; \mathbf{k} \uparrow, \mathbf{k}^{\prime} \downarrow\right)=\frac{2 \pi}{\hbar}\left|V\left(\mathbf{q}, \varepsilon_{\mathbf{k}+\mathbf{q}}-\varepsilon_{\mathbf{k}}\right)\right|^{2} .
$$

There is an equal probability that an electron scatters off another electron with equal or opposite spin, so one can sum over the Eqs. (3) and (4) to obtain an "average" scattering probability $^{9}$

$$
\begin{aligned}
\bar{w}(\mathbf{k}+ & \left.\mathbf{q}, \mathbf{k}^{\prime}-\mathbf{q} ; \mathbf{k}, \mathbf{k}^{\prime}\right) \\
= & \frac{2 \pi}{\hbar}\left\{\left|V\left(\mathbf{q}, \varepsilon_{\mathbf{k}+\mathbf{q}}-\varepsilon_{\mathbf{k}}\right)\right|^{2}-\frac{1}{2} \operatorname{Re}\left[V\left(\mathbf{q}, \varepsilon_{\mathbf{k}+\mathbf{q}}-\varepsilon_{\mathbf{k}}\right)\right.\right. \\
& \left.\left.\times V^{*}\left(\mathbf{k}^{\prime}-\mathbf{q}-\mathbf{k}, \varepsilon_{\mathbf{k}^{\prime}-\mathbf{q}}-\varepsilon_{\mathbf{k}}\right)\right]\right\} .
\end{aligned}
$$

The first and second terms in Eq. (5) are referred to as the direct and exchange terms, respectively.

In practice, the exchange term often makes calculations considerably more complicated and is usually ignored. The physical grounds for doing so are as follows. First, $e-e$ collisions are usually dominated by small $q$ scattering [because $V(q, \varepsilon)$ falls quickly with $q$ for finite $\varepsilon]$. The direct (exchange) term has the form $|V(q, \varepsilon)|^{2}\left[V(q, \varepsilon) V\left(q^{\prime}, \varepsilon^{\prime}\right)\right.$ with $q^{\prime} \gg q$ ], which implies that the direct term usually dominates over the exchange. Also, the sign of exchange term can sometimes be negative, which leads to cancellation of this term within the collision integral. The effect of the exchange term was studied in a 3D system with statically screened interaction. ${ }^{10}$ There it was found that the exchange term was significant for $n a_{0}^{3}=1$ but not for $n a_{0}^{3}=0.1$. In the calculations that follow we have used $n a_{0}^{2}=0.15$, and since we furthermore include dynamical screening, which leads to a peaked interaction at small $q$, we can assume that the direct interaction dominates in our case.

We write down the formal expressions for the electronelectron scattering operator both including and excluding the exchange term. However, in the actual numerical evaluation, we ignore the exchange interaction.

\section{ELECTRON-ELECTRON SCATTERING OPERATOR}

The Boltzmann equation for electrons in uniform electric field $\mathbf{E}$ producing a force $\mathbf{F}=(-e) \mathbf{E}$ is

$$
\hbar^{-1} \mathbf{F} \cdot \frac{\partial f}{\partial \mathbf{k}}=\left(\frac{\partial f}{\partial t}\right)_{e-e}+\left(\frac{\partial f}{\partial t}\right)_{p, i},
$$

where the subscripts $e-e$ and $p, i$ are for scattering due to electron-electron interactions and the phonon+impurity interactions, respectively.

We define the function $\Psi(\mathbf{k})$, related to the deviation of the distribution function from equilibrium, as

$$
f(\mathbf{k})-f_{0}(k) \equiv f_{0}(k)\left[1-f_{0}(k)\right] \Psi(\mathbf{k}) .
$$

This function can be written in terms of a sum of angular components

$$
\Psi(k, \theta)=\sum_{n} \psi_{n}(k) \cos (n \theta)
$$

where $\theta$ is the angle from an axis of symmetry (here, the direction of the electric field). By the assumption of cylindrical symmetry of the system, the scattering terms in the Boltzmann equation do not mix different $\cos (n \theta)$ components. ${ }^{11}$ One can therefore isolate and concentrate on the $\cos \theta \operatorname{com}$ ponent $\psi_{1}(k)$, since this is the one that affects the current and hence the mobility. In three-dimensional systems, this method was used by Rode ${ }^{12}$ for electron-phonon scattering, and extended by Sanborn $^{3}$ to include electron-electron interactions.

The $\cos \theta$ component of the linearized electron-electron collision operator [i.e., neglecting higher powers in $\Psi(\mathbf{k})$ ], which we denote $I_{e-e}\left[\psi_{1}\right]$, is ${ }^{13}$

$$
\begin{aligned}
& I_{e-e}\left[\psi_{1} x_{\mathbf{k}, \mathbf{F}}\right](k) \\
&=-2 \int \frac{d \mathbf{k}^{\prime}}{(2 \pi)^{2}} \int \frac{d \mathbf{q}}{(2 \pi)^{2}} \bar{w}\left(\mathbf{k}+\mathbf{q}, \mathbf{k}^{\prime}-\mathbf{q} ; \mathbf{k}, \mathbf{k}^{\prime}\right) \\
& \quad \times f^{0}(\mathbf{k}) f^{0}\left(\mathbf{k}^{\prime}\right)\left[1-f^{0}(\mathbf{k}+\mathbf{q})\right]\left[1-f^{0}\left(\mathbf{k}^{\prime}-\mathbf{q}\right)\right] \\
& \quad \times \delta\left(\boldsymbol{\epsilon}_{\mathbf{k}}+\epsilon_{\mathbf{k}^{\prime}}-\epsilon_{\mathbf{k}+\mathbf{q}}-\epsilon_{\mathbf{k}^{\prime}-\mathbf{q}}\right)\left[\psi_{1}(k) x_{\mathbf{k}, \mathbf{F}}+\psi_{1}\left(k^{\prime}\right) x_{\mathbf{k}^{\prime}, \mathbf{F}}\right. \\
&\left.-\psi_{1}(|\mathbf{k}+\mathbf{q}|) x_{\mathbf{k}+\mathbf{q}, \mathbf{F}}-\psi_{1}\left(\left|\mathbf{k}^{\prime}-\mathbf{q}\right|\right) x_{\mathbf{k}^{\prime}-\mathbf{q}, \mathbf{F}}\right] .
\end{aligned}
$$

Here, $x_{\mathbf{k}, \mathbf{k}^{\prime}}$ is the cosine of the angle between $\mathbf{k}$ and $\mathbf{k}^{\prime}$. The goal is to write the operator $I$ in the form

$$
I\left[\psi_{1} x_{\mathbf{k}, \mathbf{F}}\right](k)=x_{\mathbf{k}, \mathbf{F}} \int_{0}^{\infty} d p p K(k, p) \psi_{1}(p) .
$$

The kernal $K(k, p)$ is symmetric, from detailed balance, ${ }^{13}$ and the extra factor of $p$ in the integral comes from phase space. Thus, in order to incorporate electron-electron scattering for a particular density and temperature into a calculation, one need only generate $K(k, p)$ once and store it; it can then be used for all calculations involving electron-electron scattering at that density and temperature. 
The four $\psi$ 's in Eq. (9) give four terms, each of which give a contribution to the kernel, $K=K_{1}+K_{2}+K_{3}+K_{4}$. In the following subsections, we explicitly write down the form of each of these kernels.

\section{A. First term, involving $\psi_{1}(\mathbf{k})$}

The $\psi_{1}(k) x_{\mathbf{k}, \mathbf{F}}$ can be factored out, and we obtain

$$
\begin{aligned}
K_{1}(k, p)= & -\delta(k-p) k^{-1} f_{0}(k) \int \frac{d \mathbf{q}}{(2 \pi)^{2}} \\
& \times\left[1-f_{0}(\mathbf{k}+\mathbf{q})\right] 2 \int \frac{d \mathbf{k}^{\prime}}{(2 \pi)^{2}} f_{0}\left(\mathbf{k}^{\prime}\right) \\
& \times\left[1-f_{0}\left(\mathbf{k}^{\prime}-\mathbf{q}\right)\right] \bar{w}\left(\mathbf{k}+\mathbf{q}, \mathbf{k}^{\prime} ; \mathbf{k}, \mathbf{k}^{\prime}+\mathbf{q}\right) \\
& \times \delta\left(\varepsilon_{\mathbf{k}^{\prime}+\mathbf{q}}-\varepsilon_{\mathbf{k}^{\prime}}-\left\{\boldsymbol{\varepsilon}_{\mathbf{k}+\mathbf{q}}-\boldsymbol{\varepsilon}_{\mathbf{k}}\right\}\right) .
\end{aligned}
$$

In the event where the exchange interaction can be neglected, one obtains, as in Ref. 3 (we denote the scattering integral that neglects the exchange interaction with an asterisk),

$$
\begin{aligned}
K_{1}^{*}(k, p)= & -\delta(k-p)\left[\frac{f^{0}(k)}{\pi k} \int \frac{d \mathbf{q}}{(2 \pi)^{2}}\right. \\
& \times\left[1-f^{0}(\mathbf{k}+\mathbf{q})\right] \frac{2 \pi}{\hbar}\left|V\left(\mathbf{q}, \varepsilon_{\mathbf{k}+\mathbf{q}}-\varepsilon_{\mathbf{k}}\right)\right|^{2} \\
& \left.\times \operatorname{Im}\left[\chi\left(\mathbf{q}, \varepsilon_{\mathbf{k}}-\varepsilon_{\mathbf{k}+\mathbf{q}}\right)\right] n_{B}\left(\varepsilon_{\mathbf{k}+\mathbf{q}}-\varepsilon_{\mathbf{k}}\right)\right],
\end{aligned}
$$

where $n_{B}(\varepsilon)=[\exp (\beta \varepsilon)-1]^{-1}$ is the Bose function, and $\chi(q, \omega)$ is the RPA polarizability.

\section{B. Second term, involving $\psi_{1}\left(\mathbf{k}^{\prime}\right)$}

Since $\cos \left(\theta+\theta^{\prime}\right)=\cos (\theta) \cos \left(\theta^{\prime}\right)-\sin (\theta) \sin \left(\theta^{\prime}\right)$ and the sin terms vanish from symmetry considerations, we can write $x_{\mathbf{k}^{\prime}, \mathbf{F}}=x_{\mathbf{k}, \mathbf{F}} x_{\mathbf{k}^{\prime}, \mathbf{k}}$. Then, the second kernel is

$$
\begin{aligned}
K_{2}(k, p)= & -2 \frac{f^{0}(k) f_{0}(p)}{(2 \pi)^{2}} \int_{0}^{2 \pi} d \theta_{\mathbf{k}, \mathbf{p}} \cos \theta_{\mathbf{k}, \mathbf{p}} \int \frac{d \mathbf{q}}{(2 \pi)^{2}} \\
& \times w(\mathbf{k}+\mathbf{q}, \mathbf{p}-\mathbf{q} ; \mathbf{k}, \mathbf{p})\left[1-f^{0}(\mathbf{k}+\mathbf{q})\right] \\
& \times\left[1-f^{0}(\mathbf{p}-\mathbf{q})\right] \delta\left(\varepsilon_{\mathbf{k}}+\varepsilon_{\mathbf{p}}-\varepsilon_{\mathbf{k}+\mathbf{q}}-\varepsilon_{\mathbf{k}-\mathbf{q}}\right) .
\end{aligned}
$$

The $\mathbf{q}$ integration can be evaluated by the change of variables,

$$
\mathbf{q}=\mathbf{Q}+\Delta \mathbf{k} / 2, \quad \Delta \mathbf{k}=\mathbf{p}-\mathbf{k}, \quad \overline{\mathbf{k}}=(\mathbf{k}+\mathbf{p}) / 2 .
$$

Then, the $\delta$ function in Eq. (13) becomes

$$
\delta\left(\varepsilon_{\mathbf{k}}+\varepsilon_{\mathbf{p}}-\varepsilon_{\mathbf{k}+\mathbf{q}}-\varepsilon_{\mathbf{p}-\mathbf{q}}\right)=\delta\left\{\frac{\hbar^{2}}{m}\left[Q^{2}-\left(\frac{\Delta \mathbf{k}}{2}\right)^{2}\right]\right\},
$$

which gives

$$
\begin{aligned}
& K_{2}(k, p)=-\frac{m f^{0}(k) f_{0}(p)}{8 \pi^{4} \hbar^{2}} \int_{0}^{\pi} d \theta_{\mathbf{k}, \mathbf{p}} \cos \theta_{\mathbf{k}, \mathbf{p}} \\
& \times \int_{0}^{2 \pi} d \phi w(\overline{\mathbf{k}}+\mathbf{Q}, \overline{\mathbf{k}}-\mathbf{Q} ; \overline{\mathbf{k}}+\Delta \mathbf{k}, \overline{\mathbf{k}}-\Delta \mathbf{k}) \\
& \times\left[1-f^{0}\left(\varepsilon_{\overline{\mathbf{k}}+\mathbf{Q}}\right)\right]\left[1-f^{0}\left(\varepsilon_{\overline{\mathbf{k}}-\mathbf{Q}}\right)\right],
\end{aligned}
$$

where $\phi$ is the angle between $\mathbf{Q}$ and $\overline{\mathbf{k}}$.

\section{Third term, involving $\psi_{1}(|k+q|)$}

Using $x_{\mathbf{k}+\mathbf{q}, \mathbf{F}}=x_{\mathbf{k}, \mathbf{F}} x_{\mathbf{k}, \mathbf{k}+\mathbf{q}}$ in the integrand and letting $\mathbf{p}=$ $\mathbf{k}+\mathbf{q}$, gives

$$
\begin{aligned}
K_{3}(k, p)= & \frac{2 f^{0}(k)\left[1-f^{0}(p)\right]}{(2 \pi)^{2}} \int_{0}^{2 \pi} d \theta_{\mathbf{p}, \mathbf{k}} \cos \theta_{\mathbf{p}, \mathbf{k}} \\
& \times \int \frac{d \mathbf{k}^{\prime}}{(2 \pi)^{2}} f^{0}\left(\mathbf{k}^{\prime}+\mathbf{p}-\mathbf{k}\right) \\
& \times\left[1-f^{0}\left(\mathbf{k}^{\prime}\right)\right] \bar{w}\left(\mathbf{p}, \mathbf{k}^{\prime} ; \mathbf{k}, \mathbf{k}^{\prime}+\mathbf{p}-\mathbf{k}\right) \\
& \times \delta\left(\varepsilon_{\mathbf{p}}+\varepsilon_{\mathbf{k}^{\prime}}-\varepsilon_{\mathbf{k}}-\varepsilon_{\mathbf{k}^{\prime}+\mathbf{p}-\mathbf{k}}\right) .
\end{aligned}
$$

The $\delta$ function $\delta\left(\hbar^{2}(\mathbf{p}-\mathbf{k}) \cdot\left(\mathbf{k}^{\prime}-\mathbf{k}\right) / m\right)$ reduces the $d \mathbf{k}^{\prime}$ dimensional integral to one dimension.

If one neglects exchange, then as with the first term the $d \mathbf{k}^{\prime}$ integral can be done, giving

$$
\begin{aligned}
K_{3}^{*}(k, p)= & \frac{1}{2 \pi^{3}} \frac{\left[f^{0}\left(\varepsilon_{\mathbf{p}}\right)-f^{0}\left(\varepsilon_{\mathbf{k}}\right)\right]}{4 \sinh ^{2}\left[\left(\varepsilon_{\mathbf{p}}-\varepsilon_{\mathbf{k}}\right) /\left(2 k_{B} T\right)\right]} \\
& \times \int_{0}^{\pi} d \theta_{\mathbf{k}, \mathbf{p}} \cos \theta_{\mathbf{k}, \mathbf{p}} \frac{2 \pi}{\hbar}\left|V\left(\mathbf{p}-\mathbf{k}, \varepsilon_{\mathbf{k}}-\varepsilon_{\mathbf{p}}\right)\right|^{2} \\
& \times \operatorname{Im}\left[\chi\left(\mathbf{p}-\mathbf{k}, \varepsilon_{\mathbf{k}}-\varepsilon_{\mathbf{p}}\right)\right] .
\end{aligned}
$$

\section{Fourth term, involving $\psi_{1}\left(\left|\mathbf{k}^{\prime}-\mathbf{q}\right|\right)$}

The kernel is

$$
\begin{aligned}
K_{4}(k, p)= & \frac{2 f^{0}(k)}{(2 \pi)^{2}} \int_{0}^{2 \pi} d \theta_{\mathbf{p}, \mathbf{k}} \cos \left(\theta_{\mathbf{p}, \mathbf{k}}\right) \\
& \times\left(\int \frac{d \mathbf{q}}{(2 \pi)^{2}} w\left(\mathbf{k}+\mathbf{q}, \mathbf{k}^{\prime}-\mathbf{q} ; \mathbf{k}, \mathbf{k}^{\prime}\right)\right. \\
& \times\left[1-f^{0}(\mathbf{k}+\mathbf{q})\right] f^{0}(\mathbf{p}+\mathbf{q}) \\
& \left.\times \delta\left(\varepsilon_{\mathbf{k}}+\varepsilon_{\mathbf{p}+\mathbf{q}}-\varepsilon_{\mathbf{k}+\mathbf{q}}-\varepsilon_{\mathbf{p}}\right)\right)
\end{aligned}
$$

The term in the $\delta$ function goes as

$$
\varepsilon_{\mathbf{p}+\mathbf{q}}-\varepsilon_{\mathbf{p}}+\varepsilon_{\mathbf{k}}-\varepsilon_{\mathbf{k}+\mathbf{q}}=\frac{\hbar^{2}}{m}\{\mathbf{q} \cdot(\mathbf{p}-\mathbf{k})\},
$$

which reduces the $\mathbf{q}$ integration down to one dimension.

In fact, the kernels for higher-order components are very similar to the ones given above. For an angular variation proportional to $\cos n \theta$, the $K_{1}$ term is identical for all $n$, whereas with $K_{2}, K_{3}$, and $K_{4}$ one simply replaces $\cos \theta$ with $\cos n \theta$ in the $\theta$ integration. 
We have shown that one can calculate the matrix $K(k, p)$, which gives the electron-electron scattering term for small deviations from equilibrium. Once the matrix $K(k, p)$ has been calculated, one simply needs to iterate the equation for $\psi_{1}(k)$ until convergence is obtained.

In order to calculate $\psi_{1}(k)$ for the case when the $e$-e scattering rate dominates, it is often useful to use the fact that electron-electron scattering leaves a drifted Fermi-Dirac distribution invariant. ${ }^{14}$ Thus, for the case of elastic or quasielastic collisions, one can define

$$
\tilde{\psi}_{1}(k)=\psi_{1}(k)-\psi_{1}^{\mathrm{DF}}(k),
$$

where $\psi_{1}^{\mathrm{DF}}(k)=\hbar k v_{d} /\left(k_{B} T\right)$ is for a drifted Fermi-Dirac distribution. Any $v_{d}$ can be used. In our case, because we cut off the matrix $K(k, p)$ at a $k_{\max }$, which implicitly sets $\tilde{\psi}_{1}\left(k>k_{\max }\right)=0$, we chose $v_{d}$, which gives $\tilde{\psi}_{1}\left(k_{\max }\right)=0$ so that the distribution function is continuous at $k_{\max }$.

We write the linearized $e-e$ scattering term as

$$
I_{e-e}\left[\psi_{1}(k)\right]=-\frac{\psi(k)}{\tau_{e e}}+J\left[\psi_{1}(k)\right],
$$

where the first term on the right-hand side corresponds to the diagonal $K_{1}$ term and $J$ corresponds to $K_{2}+K_{3}+K_{4}$. The Boltzmann equation for $\psi_{1}(k)$ in the case when the other scattering mechanisms are quasielastic (which might include acoustic-phonon scattering, which generally involves very small energy electron loss) is

$$
\begin{aligned}
e E v(k)\left(\frac{\partial f_{0}}{\partial \varepsilon}\right)= & -\frac{f_{0}(k)\left[1-f_{0}(k)\right]\left[\tilde{\psi}_{1}(k)+\psi_{1}^{\mathrm{DF}}(k)\right]}{\tau_{\mathrm{el}}(k)} \\
& -\frac{\tilde{\psi}_{1}(k)}{\tau_{e-e}(k)}+J\left[\tilde{\psi}_{1}\right](k)
\end{aligned}
$$

where $\tau_{\mathrm{el}}^{-1}(k)$ is the quasielastic scattering rate. This implies that one must iterate the equation

$$
\tilde{\psi}_{1}(k)=\frac{\frac{e E v(k)}{k_{B} T} f_{0}(k)\left[1-f_{0}(k)\right]+J\left[\tilde{\psi}_{1}\right]-f_{0}(k)\left[1-f_{0}(k)\right] \psi_{1}^{\mathrm{DF}}(k) \tau_{\mathrm{el}}^{-1}(k)}{f_{0}(k)\left[1-f_{0}(k)\right] \tau_{\mathrm{el}}^{-1}(k)+\tau_{e-e}^{-1}(k)}
$$

to find $\psi_{1}(k)$.

\section{RESULTS AND DISCUSSION}

We study the case of electrons confined in a $100-\AA$-wide square GaAs quantum well with infinite barriers. We assume that there is a $\delta$-doping layer of (uncorrelated) charged impurities, equal in density to that of the electrons in the well, situated a distance $d$ away from the center of the well. We included three scattering mechanisms: $e-e$, chargedimpurity, and acoustic-phonon scattering, and we approximated the acoustic-phonon scattering as being elastic.

We calculated the matrix in the form of $200 \times 200$ grid points from $E=0$ to $E=5 k_{F}$, and we used spline routines to interpolate between the grid points. The $K_{3}(k, p)$ and $K_{4}(k, p)$ diverge logarithmically as $k \rightarrow p$, which complicates the splining procedure, but we got around this problem by splining $K_{3,4}(k, p) / \ln (|k-p|)$, which is a smooth function.

In Fig. 1, we show the deviation function $\psi_{1}(k) / k$ for a fixed density $n=1.5 \times 10^{11} \mathrm{~cm}^{-2}$ and temperature $T=30 \mathrm{~K}$, for several different distances $d$ of the ionized impurities from the center of the quantum well. Note that when the distribution is a drifted Fermi-Dirac function, $\psi_{1}(k) / k$ $=$ const. Thus, as the impurities are moved further away, the impurity scattering becomes weaker and the $e-e$ scattering starts to dominate ${ }^{15}$ and drives the distribution function closer to a drifted Fermi-Dirac function. The inset shows $\psi_{1}(k)$ calculated both including and excluding electronelectron scattering for $d=100 \AA$, which shows more clearly the effect of $e-e$ on $\psi_{1}(k)$. While transport experiments in a single layer are not particularly dependent on the details of the shape of $\psi_{1}(k)$, it has been shown ${ }^{7}$ that drag experiments in coupled quantum wells are quite sensitive to the details of $\psi_{1}(k)$. In particular, when $\psi_{1}(k)$ rises faster than $k$ (which implies that there are more carriers in the high-energy region than for a drifted Fermi-Dirac distribution), the drag rate

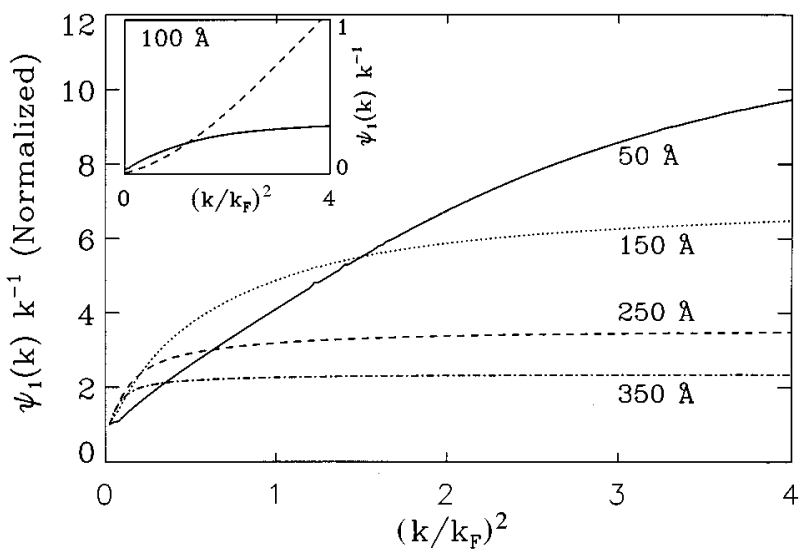

FIG. 1. Deviation function $\psi_{1}(k) k^{-1}$ (normalized to 1 at $k=0$ ) for electrons responding to a weak static electric field in a GaAs quantum well, width $100 \AA$, density $n=1.5 \times 10^{11} \mathrm{~cm}^{-2}$ and temperature $T=30 \mathrm{~K}$, for distances $d=50,150,250$, and $350 \AA$ of the charged impurity layer from the center of the well. The collision term includes screened impurity, acoustic-phonon and electronelectron scattering terms. The further the impurities are moved away from the well, the more dominant the electron-electron scattering becomes and the distribution tends to a drifted Fermi-Dirac (a pure drifted Fermi-Dirac is a straight horizontal line). The solid (dashed) curve in the inset shows the deviation function (in arbitrary units) for $d=100 \AA$ calculated including (excluding) electronelectron scattering. 


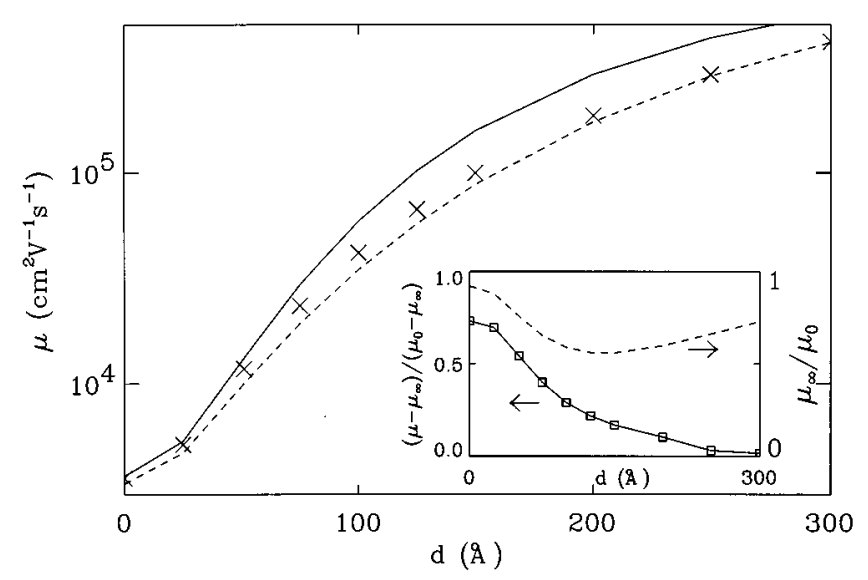

FIG. 2. Mobility as a function of impurity distance from the center of the well. Parameters as in Fig. 1. Crosses are for $\mu$ calculated from the Boltzmann equation, solid line is for $\mu_{0}$ (no $e$ $e$ ) and dashed line is for $\mu_{\infty}$ (infinite $e-e$ ). Inset: (i) $\left(\mu-\mu_{\infty}\right) /\left(\mu_{0}-\mu_{\infty}\right)$ as a function of impurity distance from center of well (solid line). As the mobility passes through $\sim 3 \times 10^{4}$ $\mathrm{cm}^{2} \mathrm{~V}^{-1} \mathrm{~s}^{-1}$, where $e-e$ and impurity scattering rates are approximately comparable, the mobility crosses over from $\mu_{0}$ to $\mu_{\infty}$. (ii) The ratio $\mu_{\infty} / \mu_{0}$ (dashed line). This ratio can be as small as $\approx 0.5$.

increases because high-energy particles give a larger contribution to the overall drag rate, and there is greater opportunity for coupling to the plasmons of the system, which also enhances the drag rate. Therefore, for the purpose of calculating the drag rate in coupled quantum wells in intermediate temperatures, it is crucial to calculate the actual form of $\psi_{1}(k)$ accurately, including all salient scattering mechanisms.

Figure 2 shows the mobility $\mu$ as a function of ionized impurity distance $d$ from the center of the quantum well. Also shown are the mobilities $\mu_{0}=e\langle\tau\rangle / m$ and $\mu_{\infty}=e /\left(\left\langle\tau^{-1}\right\rangle m\right)$, for the limits of zero and infinite electronelectron scattering, respectively. The $\mu_{0}$ is generally larger than $\mu_{\infty}$ because $e-e$ scattering tends to scatter "runaway" electrons with large velocities (where the impurity scattering rate is small) back into lower velocity states. The inset shows that $\mu_{0}$ for this case can be almost twice $\mu_{\infty}$.

As $d$ becomes larger, the $e-e$ scattering dominates over all other scattering mechanisms and $\mu \rightarrow \mu_{\infty}$. Conversely, for small $d$, the impurity scattering is relatively large compared to the electron scattering, and $\mu$ is closer to the $\mu_{0}$ than $\mu_{\infty}$. The crossover from $\mu_{0}$ to $\mu_{\infty}$ is shown with the open squares in the inset of Fig. 2 . The crossover occurs when the impurity scattering and electron-electron mean free paths become equivalent. The transport scattering rate, for impurity scattering is given by $\tau_{\mathrm{tr}}^{-1}=e /(\mu \mathrm{m})=2.6 \times 10^{16}$ $\mathrm{cm}^{2} \mathrm{~V}^{-1} \mathrm{~s}^{-2} / \mu$. The electron-electron scattering in twodimensional systems is approximately given by ${ }^{16,17}$

$$
\tau_{e-e}^{-1} \approx \frac{E_{F}}{\hbar}\left[\frac{k_{B} T}{E_{F}}\right]^{2}\left[\ln \left(\frac{E_{F}}{k_{B} T}\right)+\ln \left(2 \frac{q_{T F}}{p_{F}}\right)+1\right] .
$$

For this system, this is on the order of $10^{12} \mathrm{~s}^{-1}$. Thus, the crossover point, which should occur when $\tau_{\text {tr }}^{-1}$ and $\tau_{e-e}$ are equal, is given by $\mu \approx 3 \times 10^{4} \mathrm{~cm}^{2} \mathrm{~V}^{-1} \mathrm{~s}^{-1}$. An inspection of Fig. 2 shows this to hold. Chabasseur-Molyneux et $a .^{2}$ have also experimentally found this crossover in $\mathrm{GaAs} / \mathrm{Al}_{x} \mathrm{Ga}_{1-x} \mathrm{As}$ heterojunctions. Finally, at $d=150 \AA$, $\mu \approx 0.6 \mu_{0}$, implying the $e-e$ scattering has caused a substantial reduction in the mobility.

To summarize, in this paper we have described a method of including electron-electron scattering, including full finite-temperature dynamical screening, exactly in the Boltzmann equation, for small deviations of the distribution function from equilibrium. Using this method to calculate the distribution function and mobilities for electrons in a GaAs quantum well, we find a well-defined crossover from $\mu_{0}$ to $\mu_{\infty}$ (which can be significantly different from each other) when the $e-e$ and impurity scattering mean free paths are equivalent. For certain parameters studied, $e-e$ is responsible for reduction in the mobility of up to $40 \%$.

\section{ACKNOWLEDGMENTS}

We thank Karim El-Sayed for useful discussions. K.F. was supported by the Carlsberg Foundation.
*Electronic address: benhu@mic.dtu.dk

†Electronic address: KAF@kelvin.dfm.dtu.dk

Present and permanent address.

${ }^{1}$ L. W. Molenkamp and M. J. M. de Jong, Phys. Rev. B 49, 5038 (1994)

${ }^{2}$ V. Chabasseur-Molyneux et al., Phys. Rev. B 51, 13793 (1995).

${ }^{3}$ B. A. Sanborn, Phys. Rev. B 51, 14256 (1995).

${ }^{4}$ W. W. Schulz and P. B. Allen, Phys. Rev. B 52, 7994 (1995).

${ }^{5}$ C. Herring and E. Vogt, Phys. Rev. 101, 944 (1956).

${ }^{6}$ R. W. Keyes, J. Phys. Chem. Solids 6, 1 (1958).

${ }^{7}$ K. Flensberg and B. Y.-K. Hu, Phys. Rev. B 52, 14796 (1995).

${ }^{8}$ K. El-Sayed, T. Wicht, H. Haug, and L. Bányai, Z. Phys. B 86, 345 (1992).

${ }^{9}$ The $\left|V\left(\mathbf{k}^{\prime}-\mathbf{q}-\mathbf{k}, \varepsilon_{\mathbf{k}^{\prime}-\mathbf{q}}-\varepsilon_{\mathbf{k}}\right)\right|^{2}$ in Eq. (3) term has been transformed to the $\left|V\left(\mathbf{q}, \varepsilon_{\mathbf{k}+\mathbf{q}}-\varepsilon_{\mathbf{k}}\right)\right|^{2}$ term by a change of variables, $\mathbf{q}^{\prime}=\mathbf{k}^{\prime}-\mathbf{q}-\mathbf{k}$. This transformation leaves the product of other terms in the electron-electron scattering integral invariant.

${ }^{10}$ J. Collet, Phys. Rev. B 47, 10279 (1993).
${ }^{11}$ An analogy can be made with rotationally invariant twodimensioinal systems in quantum mechanics, where angular momentum eigenstates have the form $\exp (\operatorname{in} \theta)$. Rotationallyinvariant operators commute with the angular momentum operator, and hence do not mix eigenstates of different angular momenta. In our problem, the scattering term corresponds to the rotationally-invariant operator and the $\psi_{n}(k) \cos (n \theta)$ correspond to superpositions of angular momentum eigenstates $\exp (\operatorname{in} \theta)+\exp (-i n \theta)$.

${ }^{12}$ D. L. Rode, in Semiconductors and Semimetals, edited by R. K. Willardson and A. C. Beer (Academic, New York, 1975), Vol. 10, Chap. 1.

${ }^{13}$ H. Smith and H. H. Jensen, Transport Phenomena (Clarendon Press, Oxford, 1989).

${ }^{14}$ Seeing if a drifted Fermi-Dirac distribution gives zero in $(\partial f / \partial t)_{e-e}$ is in fact a useful test of one's numerical procedure.

${ }^{15}$ The acoustic-phonon scattering is much weaker than $e$-e scattering and comparable with the impurity scattering only for $d \approx 800 \AA$. 
${ }^{16}$ G. F. Giuliani and J. J. Quinn, Phys. Rev. B 26, 4421 (1982).

${ }^{17}$ Strictly speaking, a "transport" $e-e$ scattering rate (as opposed to lifetime rate), equivalent to the impurity transport scattering rate, should be computed in order to make the comparison between the two. While the transport time merely involves an extra factor of $(1-\cos \theta)$ for impurities, it is not so simple for $e-e$ because of the complex structure of the collision integral. Hence, for heuristic purposes, we use the lifetime, which seems to give the right order of magnitude in this case. 